\title{
Dulce Ma. Gillbón, Saniya Majmutova, Sabine Pfleger y Chiara Donà [eds.] Lenguas y Diseño: Puntos de encuentro. México, CELE-UNAM, 2005.
}

En la actualidad existen en el mercado editorial una gran variedad de textos y materiales didácticos para la enseñanza de idiomas, aunque no siempre éstos responden a las necesidades particulares de alumnos, profesores e instituciones. Razón por la cual la revisión y lectura del título Lenguas y Diseño: Puntos de encuentro resulta atractiva.

El libro pertenece a la serie "Líneas de Investigación en el Departamento de Lingüística aplicada" del Centro de Enseñanza de Lenguas Extranjeras (CELE) de la UNAM en este volumen encontramos compiladas 17 experiencias de especialistas en la enseñanza de lenguas.

A decir de las editoras, Dulce Ma. Gilbón, Saniya Majmutova, Sabine Pfleger y Chiara Donà la lectura de esta obra va dirigida: al profesor de lenguas para mejorar su práctica docente y su formación académica; al diseñador que desea experimentar la incorporación de nuevos enfoques metodológicos y nuevas tecnologías; al formador de profesores en busca de un texto capaz de integrar metodologías de la enseñanza con el diseño de programas y materiales; al lingüista aplicado interesado en los procesos y fundamentos teóricos del diseño curricular y de materiales para distintos soportes y por último, al investigador de otras áreas disciplinadas con preguntas respecto a la interrelación entre el diseño curricular y el desarrollo de materiales de lenguas

El libro se encuentra organizado en cuatro secciones:

1. Consideraciones sobre el diseño para la enseñanza de lenguas integrada por dos artículos, uno de ellos elaborado por Dulce María Gilbón, Saniya Majmutova, Sabine Pfleger y Chiara Donà, quienes dan a conocer el trabajo que desde su fundación el CELE, ha desarrollado en el diseño curricular y materiales siempre atendiendo a las necesidades lingüistico-comunicativas, académicas y socio afectivas de su comunidad estudiantil.

El segundo trabajo reúne la experiencia de Brian Tomlinson experto en el diseño de materiales, en su trabajo presenta la propuesta de elaboración de materiales a partir de dos marcos de referencia diferentes: el texto, el cual sirve para producir libros de enseñanza presencial y la actividad que se enfoca en el desarrollo de tareas y actividades que promueven el aprendizaje autónomo.

2. Temas específicos en el diseño de materiales, en esta sección encontramos textos que hablan acerca del autoaprendizaje dirigido. Expuesto por Ma. del Rosario Aragón y Adelia Peña 
quienes explican que los centros de autoacceso surgen como una alternativa para el aprendizaje de idiomas, que su estructura académica está compuesta por asesores quienes ayudan a los estudiantes en su proceso de aprendizaje y que los materiales didácticos que son un apoyo para los estudiantes deseosos de aprender un idioma de manera autodidacta, motivo por el cual su elaboración requiere de alternativas metodológicas adecuadas, que las autoras mencionan.

El siguiente tema a tratar en esta sección es el referente a la Evaluación. Chiara Donà y Sabine Pfleger nos dan a conocer un fragmento de su investigación denominada "Modelo de evaluación comunicativa" sobre cómo mejorar la competencia oral de los estudiantes de la lengua alemana.

Por otro lado, el tema de la Gramática es abarcado en dos artículos uno realizado por Marilyn Back y Diana Jenkins y otro elaborado por Saniya Majmutova, en ambos trabajos las autoras nos informan sobre su experiencia en el estudio y práctica del método de Van Setter utilizado para la elaboración de material didáctico.

En este mismo tema - GramáticaRosa Esther Delgadillo profundiza en uno de los problemas fundamentales en la enseñanza de lenguas, que es el de no contar con materiales didácticos idóneos que auxilien a los estudiantes en el desarrollo de las habilidades lingüísticas y comunicativas compartiéndonos su experiencia en la elaboración de los nuevos materiales de español en el Centro de Enseñanza para Extranjeros (CEPE).

Para concluir este apartado, Franca Bizzoni, Liony Mello y Anna Ma. Satta presentan el contenido de los títulos que integran la serie Cuadernos gramaticales complementarios a los cursos de italiano, una propuesta de ejercicios que permitirán a los usuarios tener acceso a una cultura distinta y ampliar sus conocimientos en diferentes áreas.

3. E1 diseño de materiales y los medios, el uso de los diferentes medios de comunicación resulta una herramienta útil para la enseñanza en general y como una herramienta imprescindible en la enseñanza de otro idioma ejemplo de ello es la ponencia de Marina Chávez, Laura Cisneros, Ma. Del Rosario Aragón, Laura San Juan y Osmán Lucero quienes muestran una semblanza del proceso de elaboración de un CD de canciones para la práctica de la lengua italiana.

Y que decir del uso de Internet en la enseñanza Lucía Cámara con su trabajo pretende motivar a los profesores de francés para hacer uso de algunos sitios pedagógicos, ayudando de esta manera a desarrollar en el estudiante la autonomía en el proceso de enseñanza-aprendizaje. Martha Rico y Dulce Ma. Gilbón exponen las actividades interactivas desarrolladas en diversos cursos en línea. Leticia Martinek, Ma. del Carmen Contijoch y Martha Rico, en este artículo nos narran sus vivencias en el diseño didáctico de los diferentes módulos del diplomado en línea "Actualización en lingüística aplicada a distancia para profesores de lenguas", por último, Dulce Ma.Gilbón y Ma.Antonieta Rodríguez describen su experiencia en la participación en el diseño del sitio de consulta ILA: Curso de introducción a la lingüística aplicada. 
Por otro lado, Marina Chávez y Luis Cabrera nos exponen el proceso de conceptualización y metodología que emplearon para la producción de una serie de televisión denomina Lets's listen: inglés con cine, música y algo más.

Para terminar Jesús Valdez, Yumiko Hocino, Kazuko Nagao y Kazuko Hozumi nos convidan de su experiencia en el diseño y producción de cuatro programas de video que promueven el uso adecuado de los diccionarios kanjis y japonésespañol, así como también un manual de ejercicios.

4. Diseño curricular es la última sección del libro compuesta por dos trabajos, uno presentado por Noemí Alfaro, Eréndira Camarena, Leonardo Herrera, Clara Martínez, Paloma Mora y Juan A. Ortiz integrantes de un proyecto que elabora material didáctico para la enseñanza del portugués, dirigido a estudiantes universitarios basado en las necesidades de los alumos y profesores usuarios del mismo. Finalmente, Dulce Ma. Gibón, Karen Lusnia, Rosa Esther Delgadillo y Ma. del Carmen Contijoch miembros de un grupo multidisciplinario de académicos de la UNAM, concientes de la necesidad de contar con profesionales en la enseñanza de la lengua expresan tanto las consideraciones teóricas, metodológicas y operativas necesarias para la creación del diplomado en línea "Actualización en lingüística aplicada a distancia para profesores de lenguas" actualmente en operación.

La lectura completa o aleatoria de las experiencias presentadas en la obra, nos brinda la oportunidad de enriquecer o conocer nuestros conocimientos en el campo de la enseñanza de una segunda lengua.

Ariadna Vaca

CEPE-UNAM 
